\title{
ANN-based Classification of Operating Data in Humid Air-Water Heat Exchangers
}

\author{
Gabriela Avila ${ }^{1}$, Arturo Pacheco-Vega ${ }^{2}$ \\ ${ }^{1}$ Universidad Autonoma de San Luis Potosi \\ San Luis Potosi, SLP 78210, Mexico \\ gaby.avila.rosa@gmail.com \\ ${ }^{2}$ California State University, Los Angeles \\ Los Angeles, CA 90032, USA \\ apacheco@calstatela.edu
}

\begin{abstract}
In this paper we present a classification algorithm, based on artificial neural networks, which can be used to allocate data heat exchangers undergoing possible condensation into the appropriate operating conditions. The neural network has a feedforward fully connected architecture and uses the typical backpropagation algorithm to adjust its weights during training. The ANN classification methodology is grounded on the idea that, common patterns in data sets used during training and testing give rise to small prediction errors. This idea then provides the discrimination criteria that enable data allocation into pre-established groups. The results from the application to published condensing heat exchanger data show that the ANN-based methodology is able to accurately identify the data corresponding to each of the specified conditions in the system, as shown via independent assessment using a related cluster analysis technique. This methodology has the potential to be an alternative to visual techniques as a pattern recognition tool for complex physical phenomena occurring in thermal systems.
\end{abstract}

Keywords: Heat exchangers; Artificial neural networks; Data classification.

\section{Introduction}

Heat exchangers are common in industrial applications, and for their design and control it is necessary to estimate their performance. Such evaluation is complicated from a mathematical viewpoint due to complexities arising from both geometry and the occurring phenomena. Thus, the common practice is to develop empirical models based on correlation equations from specific prototypes, and use them later to perform the thermal design calculations. These nondimensional correlations, which are derived from specific prototypes, effectively compress experimental information about the heat exchanger operation into two heat transfer coefficients. From these quantities, the heat transfer rate can later be obtained. A major drawback of the compression process, however, is that often the resulting models generate large errors in their predictions [1]. Although some recent studies have found a number of factors leading to such prediction errors, which include the nonuniqueness of the correlation constants [2], the assumptions in the analysis [3], and the functions used in the correlation equations [4], another factor that plays a significant role is the data classification process.

Correlation equations are built from experimental data commonly classified by visual methods into the corresponding physical conditions. However, despite technological progress in visualization techniques [5], they still generate large uncertainties, particularly in cases when two phase flows are present, as it is the case in condensing heat exchangers. To address this issue, we have recently proposed the use of cluster analysis to algorithmically classify condensing heat exchanger data, and the results from it have been reported elsewhere [6, 7].

This work focuses on a methodology based on artificial neural networks (ANNs), to classify performance data heat exchangers under possible condensing conditions. To this end, we first describe the heat exchanger data used in the analysis, and then illustrate - in some detail - the ANN-based classification technique. Later, the methodology is applied to allocate the experimental data are classified into three groups, roughly resembling the typical conditions of dry-surface, dropwise and film condensation. Finally, the results are compared to those from the visual procedure used by McQuiston [8], and to those obtained by our clustering methodology [6,7], illustrating the accuracy of the ANN classification. 


\section{Experimental Data}

The experimental data used here were collected and published by McQuiston [8] from tests on a number of multiplerow multiple-column fin-tube heat exchangers, with staggered tubes. Their geometry, of nominal size of $127 \mathrm{~mm} \times 305$ $\mathrm{mm}$, with varying fin spacing, is schematically shown in Figure 1. These data, which are depicted in Figure 2 via cut planes, and the correlations derived from them [9], are standards in HVAC applications. The experiments were carried out with atmospheric air flowing through the fin passages and water flowing inside the tubes of the device, under a wide range of operating conditions, including possible condensation on the fins. Whether the fins were dry, or covered with water droplets or water film, was determined by direct observations and recorded. The total number of measurements was 327, of which 91 were classified as dry surface, 117 as dropwise condensation, and 119 as film condensation. Importantly, since the study focused on the external side of the heat exchanger, high Reynolds-number turbulent flow on the water side was used to decouple the two thermal resistances. Thus, the air-side transfer coefficient was given in terms of the Colburn $j$-factor.

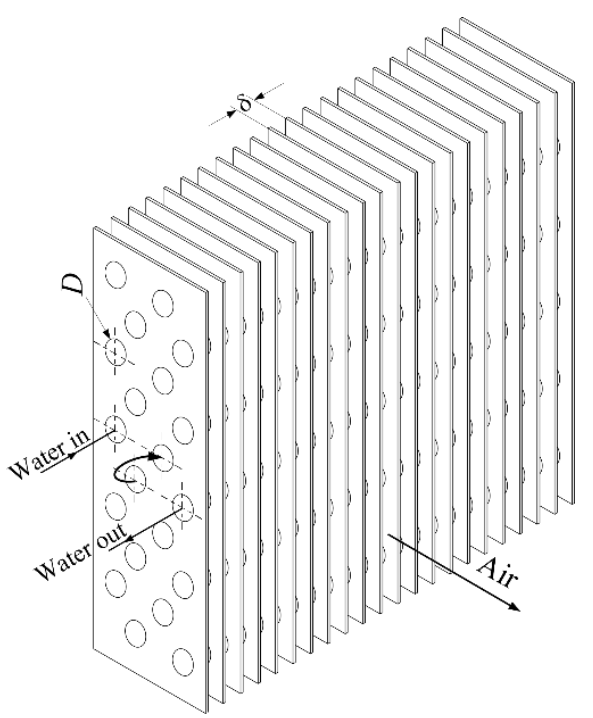

Fig. 1: Schematic of compact heat exchanger.

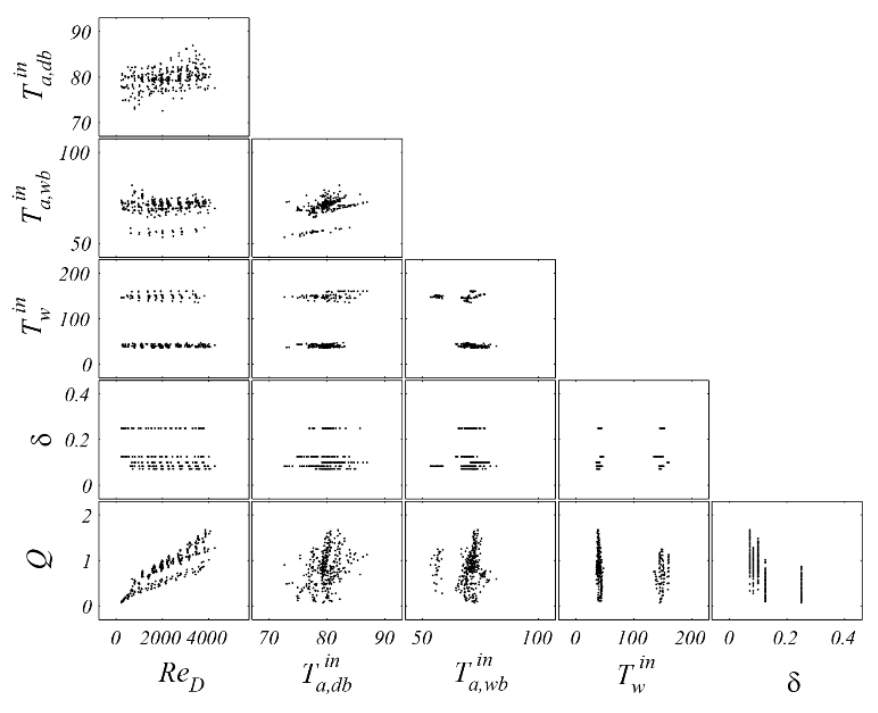

Fig. 2: Cut-plane data representation.

The variables reported in [8] include: the flow rate of humid air as Reynolds numbers, $R e_{D}$, the dry-bulb and wetbulb inlet air temperatures, $T_{a, d b}^{i n}$ and $T_{a, w b}^{i n}$, the fin spacing, $\delta$, the inlet water temperature, $T_{w}^{i n}$, and the heat transfer rate, $\dot{Q}$, which was computed via enthalpy balances in the external-side of the device, between its inlet and outlet ports. The corresponding ranges in operating conditions are [8]: $R e_{D} \in[220,4266], \quad T_{a, d b}^{i n} \in[73,87]^{\circ} \mathrm{F}, T_{w, d b}^{\text {in }} \in$ $[53,82]^{\circ} \mathrm{F}$, with five values of fin spacing $\delta \in\{0.25,0.125,0.10,0.083,0.0714\}$ in, $T_{w}^{\text {in }} \in[35,160]^{\circ} \mathrm{F}$, and the heat rates in $\dot{Q} \in[700,16700] \mathrm{BTU} / \mathrm{h}$.

For the aim of this study, the structure of the data can be observed in Figure 2, through a series of plane cuts passing through the six-dimensional hyper-surface $\dot{Q}=\dot{Q}\left(R e_{D}, T_{w}^{i n}, T_{a, d b}^{i n}, T_{a, w b}^{i n}, \delta\right)$, where the information is presented in as an array, with each of the five variables being plotted against all others. Although only partial information is obtained this way, it gives an idea of the interplay among the variables and the possible groups in which they can be classified. From the figure it can be seen that, in some planes, the data sorting can be easily performed (e.g., two groups exist for $T_{w}^{i n}$, as dependent variable), but such grouping is difficult in others (e.g., either one, two or more groups exist for $\dot{Q}$ as dependent variable). 


\section{Classification Methodology}

An alternative approach to the visual data classification is the methodology presented next, which is based on artificial artificial neural networks.

\section{1. Feedforward Artificial Neural Networks}

The artificial neural network (ANN), which is rooted in the biological network of the brain to replicate its functionality, is undoubtedly the most popular technique in the field of artificial intelligence (AI). ANNs "learn" from external experience in a supervised manner, and have been used in applications where some type of pattern recognition (or classification process) was needed. Within the context of thermal engineering, ANNs have been used for prediction and control of heat exchangers $[10,11]$, among other applications. Though various kinds of ANNs exist, the feedforward architecture is common in engineering applications, and it is the type of network used here to develop the classification methodology. Following Pacheco-Vega et al. [12], a feedforward ANN model of the heat exchanger at hand is schematically illustrated in Figure 3. This configuration has one input layer build with five nodes, two hidden layers, with five and three nodes respectively, and one output layer with a single node. Since the heat rate Q, is a function of inlet temperatures, flow rates and the geometry, then the input variables are: $R e_{D}, T_{w}^{i n}, T_{a, d b}^{i n}, T_{a, w b}^{i n}$ and $\delta$, whereas $\dot{Q}$, is the output variable. Details about mathematical background and procedures for training and testing the ANN, as well as an account of its history, are in Haykin [13].

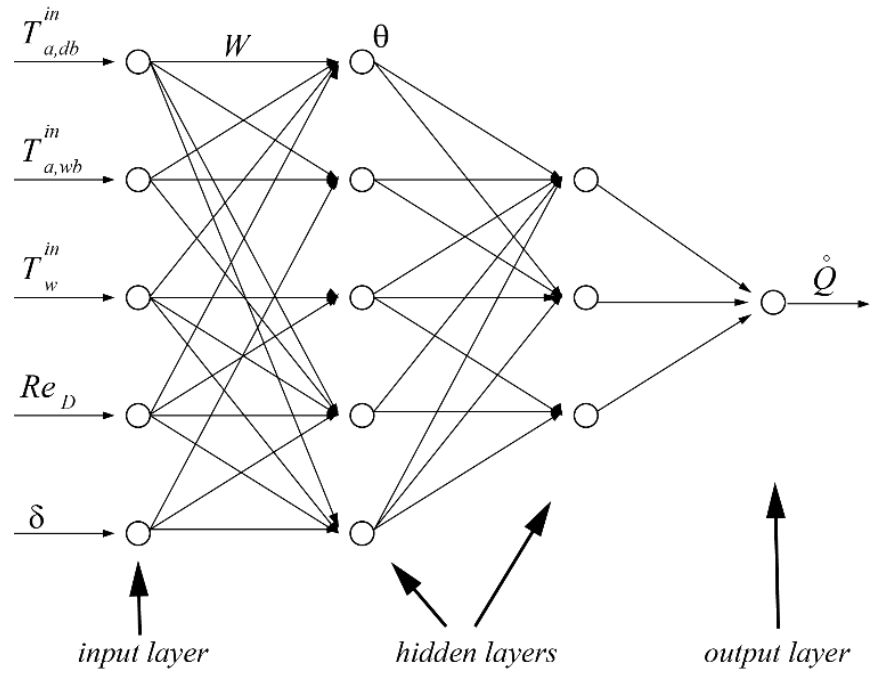

Table 1: ANN classification of heat exchanger data.

\begin{tabular}{|c|c|c|c|}
\hline Condition/Group & A & B & C \\
\hline Dry surface & $100 \%$ & 0 & 0 \\
\hline Drop condensation & 0 & $34.19 \%$ & $65.81 \%$ \\
\hline Film condensation & 0 & $21.85 \%$ & $78.15 \%$ \\
\hline
\end{tabular}

Table 2: Clustering classification of heat exchanger data $[6,7]$.

\begin{tabular}{|c|c|c|c|}
\hline Condition/Group & A & B & C \\
\hline Dry surface & $100 \%$ & 0 & 0 \\
\hline Drop condensation & 0 & $35.89 \%$ & $64.11 \%$ \\
\hline Film condensation & 0 & $25.21 \%$ & $74.79 \%$ \\
\hline
\end{tabular}

Fig. 3: A 5-5-3-1 ANN for the heat exchanger.

The ANN classification methodology developed here is grounded on the idea that given two datasets - one for training and the other for testing - if there exist common patterns in them, the ANN model build from the training set will provide a small prediction error on the testing set; otherwise, the prediction error will be large. A similar idea was used by PachecoVega et al. [14], in the context of reliability of ANN predictions under limited amount of data. For the problem at hand, the prediction error from a trained ANN can be quantified in terms of the percentage difference between the target values and the ANN predictions of the heat rate, $\dot{Q}$, as

$$
E_{p, i}=\left(\frac{\dot{Q}_{i}^{t}-\dot{Q}_{i}^{p, A N N}}{\dot{Q}_{i}^{t}}\right) \times 100, \quad i=1, \cdots, N
$$


where $\dot{Q}_{i}^{t}$ and $\dot{Q}_{i}^{p, A N N}$, for datum $i$, are the target values and the predictions, respectively. Distinguishing data that are part of a specific group from those that are not part of the set under analysis is carried out by comparing the prediction error, $E_{p, i}$, in Eq. (1), to the lower-bound error obtained during the training process of the ANN, namely, $E_{t}$, as follows:

- If $E_{p, i} \leq E_{t}$, then testing and training data points have common structures and, therefore, they belong to the same group/set.

- If $E_{p, i}>E_{t}$, then testing and training data points do not have common patterns and, therefore, they do not belong to the same group/set.

\section{2. Results and Analysis}

In order to test the ANN classification methodology, we use the 327 data sets of McQuiston [8], as classified by the visual process, and the corresponding allocation - into three groups (with group A having 91 data, whereas groups $\mathrm{B}$ and $\mathrm{C}$ have, respectively, 72 and 164 data sets) - as obtained by our cluster analysis technique [6, 7], according to the following procedure.

1. We first train three different ANNs, all having the same 5-5-3-1 configuration [12], but each with a different data set from reference [8]. For example. $\mathrm{ANN}_{1}$ is trained only with dry surface data, whereas $\mathrm{ANN}_{2}$ is trained with data of dropwise condensation and $\mathrm{ANN}_{3}$ with data corresponding to film condensation. The training errors: $E_{t}^{1}, E_{t}^{2}$, and $E_{t}^{3}$, are also recorded.

2. Then, using $\mathrm{ANN}_{1}$ and the 91 data in Group $\mathrm{A}$, the error $E_{p}^{1, A}$ is computed and compared to the corresponding training error $E_{t}^{1}$, and the data are classified according to the aforementioned procedure into data that either belong or do not belong to the training set and Group A. Similar procedures are performed with $\mathrm{ANN}_{2}$ and the 72 data from Group $\mathrm{B}$, and $\mathrm{ANN}_{3}$ with the 164 datasets of Group C.

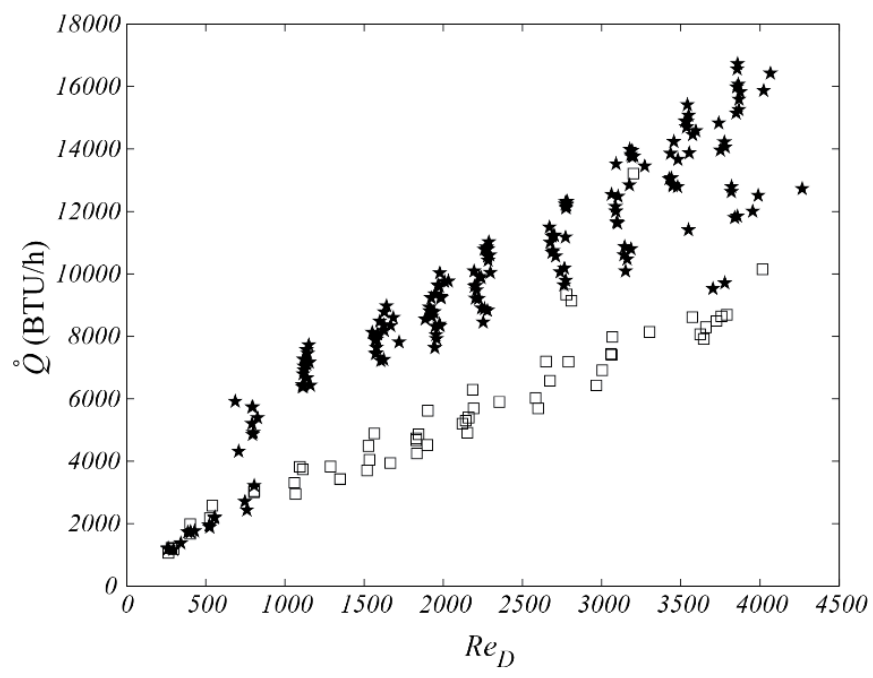

Fig. 4: ANN classification of wet-surface conditions;

Plane $\dot{Q}$ vs. $R e_{D}$. (口) Data in Group B, ( $\star$ ) Data not in group B (thus in Group C).

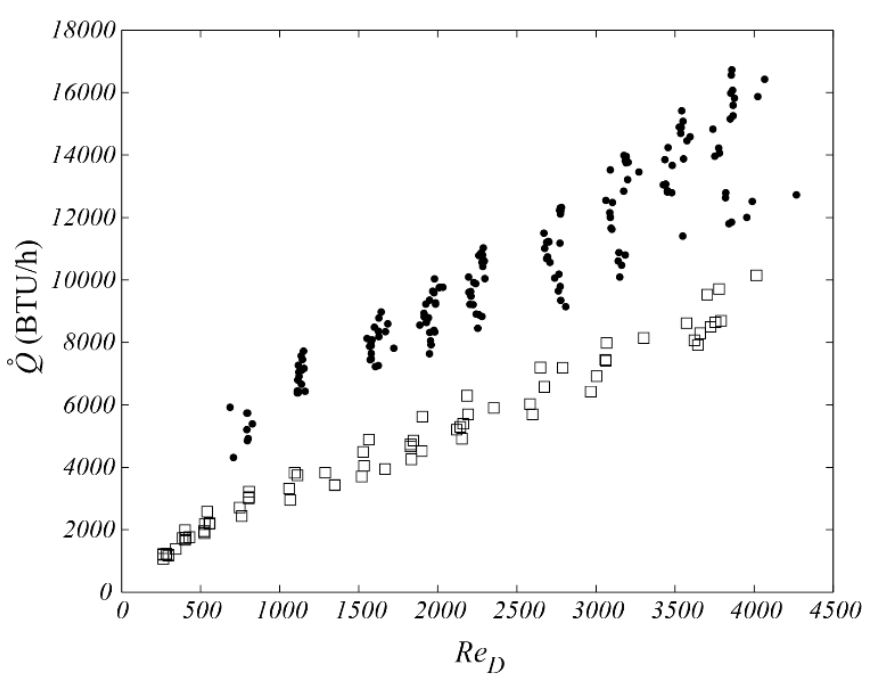

Fig. 5: Clustering classification of wet-surface conditions [6, 7]; Plane $\dot{Q}$ vs. $R e_{D} \cdot(\square)$ Group B, (•) Group C.

The classification results from the ANN-based grouping technique are shown quantitatively (as agreement in percentage between the ANN and the visual procedure of McQuiston [8]), in Table 1. Meanwhile, the allocation provided by the clustering method is shown in Table 2 . From Table 1 , it can be seen that the ANN completely agrees with the visual procedure 
for the dry-surface data, which were all classified into Group A. However, the agreement in the allocation of the data corresponding to humid conditions into Groups $\mathrm{B}$ and $\mathrm{C}$ is not as sharp, since data for drop condensation were classified into Groups B (34.19\%) and C $(65.81 \%)$, with a similar outcome for the data corresponding to film condensation. However, when comparing to the classification provided by the clustering methodology in Table 2, it can be observed that the two algorithmic groupings are in almost perfect agreement. From the two tables it is to possible to see that the complete set of data in Group A corresponds to that of dry-surface, whereas there is a difference of only $4.9 \%$ (upper-bound value) between the discrimination provided by the ANN methodology and that of the clustering technique for dropwise condensation on groups $\mathrm{B}$ and $\mathrm{C}$, and an upper-bound value of $15 \%$ between the two methodologies for film condensation on groups B and C.

Qualitatively Figures (4) and (5) show the corresponding results for wet-surface conditions using the $\dot{Q}-R e_{D}$ plane. From both figures it can be seen a very close classification obtained by both the ANN-methodology described here and the clustering technique [6,7], with only few discrepancies between them, as was also noticed from Tables 1 and 2 . These figures show that both the number and the data corresponding to each group coincide in both classification methodologies, indicating that the ANN-based technique is very accurate in classifying the different surface conditions. The disagreement with the visual classification is indicative of the complexity of the phenomenon involved in the transfer of heat in the thermal system.

\section{Conclusion}

In the current work we have introduced an algorithm based on artificial neural networks (ANNs), which is able to allocate data from condensing heat exchangers into appropriate operating conditions. The classification methodology uses a typical feedforward network architecture with backpropagation error algorithm to find patterns in the data which enable their allocation into pre-established groups. The results from the application to condensing heat exchanger data, visually classified as dry-surface, drop- and film-condensation, show that the ANN-based methodology is able to accurately identify the data corresponding to each of the conditions in the system, as shown by independent assessment using a methodology grounded on cluster analysis. Although a possible drawback of the ANN-based classification method is that the number of groups into which the data can be classified has to be known in advance, this methodology has the potential to serve as alternative to visual techniques, as a pattern recognition tool for complex physical phenomena occurring in thermal systems.

\section{Acknowledgments}

This work has been partially supported by the National Science Foundation under Award No. HRD-1547723.

\section{References}

[1] F. P. Incropera and D.P. DeWitt, Fundamentals of Heat and Mass Transfer. John Wiley \& Sons, New York, NY, 2002.

[2] A. Pacheco-Vega, M. Sen, K.T. Yang, and R.L. McClain. Genetic-algorithm-based-predictions of fin-tube heat exchanger performance. In: J.S. Lee, editor, Proceedings of the Eleventh International Heat Transfer Conference, volume 6, pages 137-142, New York, NY, August 1998. Taylor \& Francis.

[3] A. Pacheco-Vega, M. Sen, and K.T. Yang, "Simultaneous determination of in- and over-tube heat transfer correlations in heat exchangers by global regression," Int. J. Heat Mass Transfer, vol. 46, no. 6, pp. 1029-1040, 2003.

[4] W. Cai, A. Pacheco-Vega, M. Sen, and K.T. Yang, "Heat transfer correlations by symbolic regression," Int. J. Heat Mass Transfer, vol. 49, no. 23-24, pp. 4352-4359, 2006.

[5] J. A. Olivier, L. Liebenberg, J. R. Thome, and J. P. Meyer, "Heat transfer, pressure drop, and flow pattern recognition during condensation inside smooth, helical micro-fin, and herringbone tubes," Int. J. Refrigeration, vol. 30, pp. 609623, 2007.

[6] A. Pacheco-Vega and G. Avila, "Classification of condensing heat exchangers performance data by Gaussian mixtures," in Proceedings of the ASME 2009 Heat Transfer Summer Conference, San Francisco, CA, July 2009. HT2009-88627.

[7] A. Pacheco-Vega and G. Avila, "Algorithmic performance-data classification of condensing heat exchangers," Numerical Heat Transfer, Part A: Applications, DOI: 10.1080/10407782.2020.1814596, 2020.

[8] F. C. McQuiston, "Heat, mass and momentum transfer data for five plate-fin-tube heat transfer surfaces, ASHRAE Transactions, vol. 84, no. 1, pp. 266-293, 1978. 
[9] F. C. McQuiston, "Correlation of heat, mass and momentum transport coefficients for plate-fin-tube heat transfer surfaces with staggered tubes," ASHRAE Transactions, vol. 84, no. 1, pp. 294-309, 1978.

[10] A. Pacheco-Vega, "Soft computing applications in thermal energy systems." In K. Gopalakrishnan, S.K. Khaitan, and S. Kalogirou, editors, Soft Computing in Green and Renewable Energy Systems, vol. 269, pp. 1-35. Springer-Verlag, 2011.

[11] A. Pacheco-Vega, G. Diaz, M. Sen, and K. T. Yang, "Applications of artificial neural networks and genetic methods in thermal engineering." In R.P. Chhabra, editor, CRC Handbook of Thermal Engineering, chapter 4.27, pages 1217-1269. 2nd edition, 2017.

[12] A. Pacheco-Vega, G. Diaz, M. Sen, K.T. Yang, and R.L. McClain, "Heat rate predictions in humid air-water heat exchangers using correlations and neural networks" ASME J. Heat Transfer, vol. 123, no. 2, pp. 348-354, 2001.

[13] S. Haykin, Neural Networks, A Comprehensive Foundation. Prentice-Hall, Upper Saddle River, 1999.

[14] A. Pacheco-Vega, M. Sen, K.T. Yang, and R.L. McClain, "Neural network analysis of fin-tube refrigerating heat exchanger with limited experimental data," Int. J. Heat Mass Transfer, vol. 44, no. 4, pp. 763-770, 2001. 